Title: The Relative Age Effect and Success in German Elite U17 Soccer Teams Authors:

Dr. Claudia Augste

Universitätsstr. 3

D-86159 Augsburg

Phone: $0821 / 598-2814$

Fax: $0821 / 598-2828$

E-mail: claudia.augste@ sport.uni-augsburg.de

Prof. Dr. Martin Lames

Connollystr. 23

89809 München

Phone: 089/ $298-24495$

Fax: 089/ $298-24497$

E-mail: martin.lames@tum.de 


\title{
The Relative Age Effect and Success in German Elite U17 Soccer Teams
}

\begin{abstract}
The aim of the study was to examine whether there is empirical evidence for advantages in performance of soccer teams because of their relative age. The practice of selecting youth players according to their momentary performance leads to relative age effects (RAEs) that in turn stand for inefficient talent selection. We took the median of the birth dates as a measure of the effect size of the RAE and the Kolmogorov-Smirnov test to assess its significance. Birth dates in the three German U17 2008/09 First Leagues in soccer were examined (911 players). More than half of the 41 teams differed significantly from the distribution of the corresponding German cohort. There is a significant correlation between RAE and success defined by teams' final rankings (Spearman's $\rho=.328, P=.036$ ). Regression analyses reveal that with a median of birth dates one month earlier the team is expected to finish 1.035 ranks better. Accordingly, selecting early born athletes is an important aspect of success in youth soccer. However, also teams with no RAE are able to compete in the league, having the benefit to promote players with a better perspective for long and successful careers at adult age.
\end{abstract}

\section{Introduction}

The Relative Age Effect (RAE) in sport has been investigated in many studies (see Musch \& Grondin, 2001 for a review). Generally, there has been a tendency to select athletes for youth teams who are born early in the selection period. Extended research has been conducted to identify independent variables influencing the RAE. Selection level plays a role (Sherar, Baxter-Jones, Faulkner \& Russell, 2007; Mujika, 2009), physically more demanding sports are more affected by a RAE (Baxter-Jones, 1995), male athletes are more susceptible to RAE than women (Vincent \& Glamser, 
2006), and the effect diminishes as the athletes mature (Lames, Augste, Dreckmann, Görsdorf \& Schimanski, 2008). In adulthood the findings are heterogeneous: While Cobley, Schorer and Baker (2008) for example report persisting effects, RAE has sometimes been shown to disappear (Pérez Jiménez \& Pain, 2008) or, even to reverse in older athletes (Bäumler, 1998).

It has become clear that a RAE just stands for preferring early mature athletes in a selection at young age (Diamond, 1983; Baxter-Jones, 1995). In physically demanding sports, the probability of being selected because of one's physical maturity increases, non-linearly, when born early in the selection period. Although the initial advantages experienced by early-born early matures are relatively small, two selfsustaining processes may emerge which further increase differences in performance (Helsen, van Winckel \& Williams, 2005; Lames et al., 2008). First, success in sport is in general accompanied by positive feedback from parents, peers, and coaches which elicit higher motivation and self-confidence and, in turn, greater effort and better performance by the athlete. Second, additional measures for selected athletes, like extra-training with elite coaches, provide the athletes further advantages in performance which may make the athletes eligible for even further support. These positive feedback loops would, theoretically, lead to steadily increasing performance and allow for large advantages of athletes initially just born a few months earlier.

The habit of selecting early maturing athletes for youth squads is inconsistent with modern principles of talent search and development programs (Martin, Nicolaus, Ostrowski \& Rost, 1999). Coaches should look for young athletes with a potential for elite performances as adults. There is no justification for selecting according to the RAE.

The reason for this selection behavior is said to be the preference for immediate success over the long-term goals of talent promotion. As long as coaches in youth sports 
are judged according to their present success with young squads, they are well advised to select early maturing players who represent a performance advantage. Accordingly, the RAE in U17 soccer players has been a robust finding (Brewer, Balso \& Davis, 1995; Simmons \& Paull, 2001; Helsen et al., 2005; Pérez Jiménez \& Pain, 2008; Mujika et al., 2009).Vaeyens, Philippaerts \& Malina (2005) have already shown that the number of selections and minutes played vary with relative age in senior soccer on a player base.

This study aims to test whether U17 soccer teams with higher relative age are more successful than teams with more normally distributed birth dates.

\section{Methods}

\section{Sample}

In Germany top U17 teams in soccer are organized in three first leagues representing different regions (North/North-East, West, and South/South-West). At the end of each season, the winners of each league (consisting of 14 teams each) play each other to determine the national champion. Birth dates of the players from the $2008 / 09$ season were obtained either from the home pages of the 42 teams or from a website (www.transfermarkt.de) containing information on most of the players of German U17 first leagues. Squads with more than 11 players' birth dates documented were eligible for the study. According to this criterion, one team was omitted from the study. There were 911 players from 41 teams included in the study (average of 22.2 players per team). All procedures are in agreement with the ethical standards of TU München. 
In order to test the statistical significance of the RAE, we used the KolmogorovSmirnov (K-S) test. Preferentially, data from comparable populations should be used as expected distributions. In our case, the German male population born in 1992 shows a very even distribution of birth dates (median: $1^{\text {st }}$ of July; Statistisches Bundesamt Deutschland, 2008). This allows us to use an even distribution as the null hypothesis for our K-S tests.

At each birth date in the sample the empirical distribution function increases by $1 / n$ ( $n=$ sample size $)$ whereas the expected distribution function takes a value of birthday [day in the year]/365. If there is a RAE in the sample, the empirical distribution function will increase more steeply in the beginning of the year. In the K-S test, the maximum difference between the empirical and the expected distribution function is tested. For small sample sizes $(n<40)$ there are tables for critical K-S values, for larger samples there are approximations, e.g. K-S $=1.36 / V_{n}$ for $P=.05$. Figure 1 shows observed values and values expected under an equal distribution in our sample.

$* * * *$ Figure 1 near here $* * * *$

Although there are other studies employing K-S to test the significance of an RAE (e.g. Helsen et al., 2005), the most widespread practice has involved the chi-square test. Usually quarterly birthrates are compared but halves of the year and months have also been compared (recently Cobley et al., 2008; Mujika et al., 2009). The K-S test has not only the advantage of being suitable for small samples, but most importantly its degrees of freedom are free to vary with sample size. This gives the K-S test much more statistical power than the chi-square with constant degrees of freedom, (e.g. $d f=3$ ) when comparing quarters of the year. 


\section{Effect size of RAE}

In spite of the greater statistical power of the K-S test, the necessity to distinguish between effect size and rejection of hypotheses has to be considered, especially when dealing with small sample sizes (for example, analyzing single teams). We used the median of birth dates as measure for the effect size of a RAE in a sample. It directly expresses the deviation from the expected mean value of the $1^{\text {st }}$ of July in the corresponding cohort of the German population (all males born in 1992) (Statistisches Bundesamt, 2008). It is possible to calculate an analogous measure to Cohen's $d$ (Cohen, 1988) for the median and obtain effect sizes for the RAE that way. For practical reasons we used months as indicators of effect sizes for the RAE and labeled the RAEs as follows. A RAE is 'very large' if its median is before the $1^{\text {st }}$ of March. A 'large' RAE is indicated by a median in March, a median in April is termed a 'medium' effect. When the median is in May it is considered a 'small' RAE.

Characterizing the effect size of a RAE in the proposed way (large: $d \geq 0.872$, medium: $0.872>d \geq 0.588$, small: $0.588 \geq d \geq 0.303$ ) leads only to a small deviation from Cohen's $d$ (large: $d \geq 0.8$, median $\leq$ April, $8^{\text {th }}$; medium: $0.8>d \geq 0.5$, April, $8^{\text {th }} \leq$ median $\leq$ May, $9^{\text {th }}$; small: $0.5>d \geq 0.2$, May, $9^{\text {th }} \leq$ median $\leq$ June, $\left.9^{\text {th }}\right)$. Moreover, the proposed convention results in rather intuitive characterizations: a very strong RAE meaning half of the sample is born at least in the first sixth of the year, strong means that the first half is born in the first quarter, medium means born in the first third, and small means at least one month earlier than expected.

\section{Indicators of team's success}

The final rankings of the teams in the three leagues after the 2008/09 season were collected as the variable 'rank'. This variable served as the operational definition for a team's success in that season. In addition to the final ranking of a team other varia- 
bles were also taken into account. Goals for and against, and points earned during the season were extracted from the final rankings.

The relations between the strength of the RAE, (i.e. the median of the clubs' birth dates) and rank, points scored, goals for, and goals against, were measured using rank correlations. To illustrate the practical impact, the standard procedure of a linear regression analysis was used to characterize the quantitative influence of the independent variable, medians of the birth dates, on the dependent success variables mentioned above.

\section{Results}

\section{Prevalence of $R A E$}

Figure 2 shows the distribution of the birth dates of the three leagues over the months of the year. We see a strong tendency to prefer earlier born athletes and that this tendency occurs in every league with about the same strength.

$* * * *$ Figure 2 near here $* * * *$

Statistical testing revealed that there was a significant K-S test $(P<.05)$ in 22 of the 41 German U17 first league soccer teams (53.7\%). In some cases significance was not achieved because of small sample sizes.

The median of all 41 teams was the $21^{\text {st }}$ of April, which means that there was a medium RAE in the average of all clubs. The magnitude of the RAE shows the following distribution. Four teams $(9.8 \%)$ exhibited a 'very strong' RAE with a median of the birth dates in February. The largest RAE was shown by a club having half of its players born before the $20^{\text {th }}$ of February. Approximately a quarter of all teams fell into the following three categories: strong (24.4\%), medium (26.8\%) and small 
(24.4\%) RAE. Only six teams (14.6\%) did not show a RAE. The team with the smallest effect (July, 30 ${ }^{\text {th }}$ ) finished $13^{\text {th }}$ in its league of 14 clubs. Its players were born, on average, 160 days later than those of the team with the largest RAE.

\section{Impact of RAE on success}

Figure 3 shows the basic relationship examined in this study. The positive slope of the regression line indicates a positive relationship between the team's rankings in the three German first leagues and their median birth dates. Accordingly, the stronger the RAE of a team the higher its probability of ranking highly in its league.

Statistical testing of this relationship revealed a significant correlation between rank and median (Spearman's $\rho=.328, P=.036)$. A linear regression analysis $(\mathrm{F}=4.894$; $P=.033$ ) revealed that having a median birth date one month earlier is associated with an expected advantage of 1.035 ranks.

$* * * *$ Figure 3 near here****

Table I shows correlations and effect sizes between RAE and success indicators used in the study. It shows that all criteria for success in German U17 first league teams are affected in about the same way by an RAE. Clubs with a larger RAE rank higher, score more points, score more goals (although not significant) and allow fewer goals. The last column of table I gives an illustration of the practical impact of the findings. Although there are only moderate to small correlations there are considerable differences in success variables when comparing the expected values for the earliest and the latest median of the clubs' birth dates. The team with the largest RAE is expected to finish 5.5 ranks higher than the team with the smallest RAE. Furthermore, it would 
be expected to score 18.9 points and 16.6 goals more while allowing 20.3 fewer goals.

$* * * *$ Table I near here $* * * *$

\section{Discussion}

In general, RAEs are associated with many factors (Musch \& Grondin, 2001). The objective of this study was to investigate the RAE and its relationship with success in youth soccer. Subsequently, birth dates of 41 clubs of the three German U17 First Leagues were analyzed, a total of 911 players. There was at least a "medium" RAE for $61 \%$ of the clubs, defined by a median of the birth dates of its players before May, $1^{\text {st }}$, meaning that half of the team was born at least in the first third of the year. A K-S test yielded significant deviations from equal distribution in $53.7 \%$ of the teams.

These findings are in agreement with earlier studies on the RAE in soccer. A very pronounced RAE is common among male top level U17 soccer players (Brewer, Balso \& Davis, 1995; Simmons \& Paull, 2001; Helsen et al., 2005; Pérez Jiménez \& Pain, 2008; Mujika et al., 2009). Although it was not possible in this study to measure the maturity status of the players directly, we agree to the interpretation given in these studies that RAE is due to the fact that physically mature athletes have a distinct advantage in youth soccer.

We found significant relationships between the RAE and success indicators taken from final rankings for the teams in each of the leagues analyzed. There were positive correlations between the RAE and rank, points scored, and goals against. Only 
the variable 'goals scored' failed to show a significant correlation with the RAE $(P<.10)$

Comparing the expected success between the team with the largest and the smallest RAE there were remarkable differences (e.g. 5.5 ranks, 19 points scored) associated with differences in birth dates between the teams. These findings support the interpretation that success in German U17 first leagues is partially due to a higher relative age of the players which in turn means that more mature players are selected. Of course, there are many other variables influencing success such as the quality of training (Coté, Baker \& Abernethy, 2003), the club's talent promotion system and psychological traits and social support of the players (Ericsson \& Starkes, 2003).

This study has established the relationship between success and RAE. Accordingly, the RAE not only influences individual game behaviors like minutes played and number of games played for a team (Vaeyens, Philippaerts \& Malina, 2005) but also the team's overall success during the season.

But how to avoid this practice of preferring early born athletes, if they contribute to greater team success? As long as there is no agreement on taking organizational measures against the RAE, for example, changing selection dates from year to year, introducing physical development instead of or in addition to chronological age as a criterion for team membership and others (Musch \& Grondin, 2001; Helsen et al., 2005), coach education is the most often recommended measure to deal with RAEs. There are three important aspects of coach education in that sense. First, an agreement is necessary that the aim of talent promotion is to develop individual potential for elite performance at adult age. This attitude acknowledges that training in youth sport is preparatory for top level training and should be carried out accordingly. Second, as decisions for selections are unavoidable, they should focus exclusively on the perspectives a player has and less on the momentary level of performance. Third, in 
coach education an appropriate theoretical concept of talent should be introduced. Contrary to the largely prevailing notion of a genetic determination of talent this concept should emphasize the developmental potential of the players. Failing to do so may explain a great part of the findings presented in this paper.

Although these investigations need to be extended to other age groups and countries in the next paragraph some practical conclusions are mentioned.

\section{Conclusions}

The first conclusion from our findings is that selecting players with higher relative age is favorable to achieve immediate success in competition with other teams. This is in accordance with an opinion frequently held when discussing reasons for the RAE. In order to be successful in highly competitive youth sports, coaches tend to prefer older players who are physically more mature and exhibit better performance at the time of selection. Maybe due to prevailing gratification structures for coaches in youth soccer the general aim of talent promotion, to promote the most promising performers at adult age, seems to be of minor importance compared to the immediate success of their teams.

However, the corollary interpretation is more encouraging, especially for latematuring youths. Figure 3 shows that one club finished third with a negligible RAE, another team finished ninth with a median after the $1^{\text {st }}$ of July, and there are several clubs with a small RAE finishing in the first half of the league. These findings suggest that if the aim is just to compete in Germany's top leagues, it is not a necessary condition to select players with a medium, strong or very strong RAE. This in turn means that clubs may provide an excellent education to their players, including competitions against top level teams, without being victim of the RAE. The only compromise a club has to accept is that it is much less likely to achieve the top spot in 
their respective league. The benefit comes in the opportunity to promote players with a better perspective for long and successful careers in soccer.

\section{References}

Abbott, A., Button, C., Pepping, G.-J. \& Collins, D. (2005). Unnatural Selection: Talent Identification and Development in Sport. Nonlinear Dynamics, Psychology, and Life Sciences, 9, 61-88.

Bäumler, G. (1998). Der Realtivalterseffekt bei Fußballspielern und seine Wechselwirkung mit dem Lebensalter. In G. Bäumler \& G. Bauer (Hrsg.), Sportwissenschaft rund um den Fußball (S. 109-115). Hamburg: Czwalina.

Baxter-Jones, A. (1995). Growth and development of young athletes: Should competition levels be age related? Sports Medicine, 20, 59-64.

Brewer, J., Balso, P. \& Davis, J. (1995). Seasonal birth distribution amongst European soccer players. Sports, Exercise and Injury, 1, 154-177.

Cobley, S., Schorer, J. \& Baker, J. (2008). Relative age effects in professional German soccer: A historical analysis. Journal of Sports Sciences, 26, 1531-1538.

Cohen, J. (1988). Statistical Power Analysis for the Behavioral Sciences (2 ${ }^{\text {nd }}$ ed.). Hillsdale: Lawrence Erlbaum Associates.

Diamond, G. H. (1983). The Birthdate effect - a maturational effect? Journal of Learning Disabilities, 16, 161-164.

Ericsson, K. A. (Ed.). (1996). The road to excellence. The acquisition of Expert Performance in the Arts and Sciences, Sports and Games. Mahwah, NJ: Lawrence Earlbaum.

Helsen, W. F., van Winckel, J. \& Williams, M. (2005). The relative age effect in youth soccer across Europe. Journal of Sports Sciences, 23, 629-636. 
Lames, M., Augste, C., Dreckmann, C., Görsdorf, K. \& Schimanski, M. (2008). Der “Relative Age Effect" (RAE): neue Hausaufgaben für den Sport. Leistungssport, $38(6), 4-9$.

Martin, D., Nicolaus, J., Ostrowski, C. \& Rost, K. (1999). Handbuch Kinder- und Jugendtraining. Schorndorf: Hofmann.

Mujika, I., Vaeyens, R., Matthys, S. P. J., Santisteban, J., Goiriena, J. \& Philippaerts, R. (2009). The relative age effect in a professional football club setting. Journal of Sports Sciences, 27, 1153-1158.

Musch, J. \& Grondin, S. (2001). Unequal competition as an impediment to personal development: A review of the relative age effect in sport. Developmental Review, 21, 147-167.

Pérez Jiménez, I. \& Pain, M. T. G. (2008). Relative age effect in Spanish association football: Its extent and implications for wasted potential. Journal of Sports Sciences, 26, 995-1003.

Sherar, L. B., Baxter-Jones, A. D. G., Faulkner, R. A. \& Russell, K. W. (2007). Do physical maturity and birth date predict talent in male youth ice hockey players. Journal of Sports Sciences, 25, 879-886.

Simmons, G. \& Paull, G. C. (2001). Season-of-birth bias in association football. Journal of Sports Sciences, 19, 677-686.

Starkes, J. A. \& Ericsson, K. A. (2003). Expert performance in sports: Advances in research on sport expertise. Champaign, IL: Human Kinetics.

Statistisches Bundesamt, 2008. Sondertabellen aus dem Bereich der natürlichen Bevölkerungsbewegung. Wiesbaden.

Vaeyens, R., Philippaerts, R. M. \& Malina, R. M. (2005). The relative age effect in soccer : A match related perspective. Journal of Sports Sciences, 23, 747-765. 
Vincent, J. \& Glamser, F. D. (2006). Gender differences in the relative age effect among US Olympic Development Program youth soccer players. Journal of Sports Sciences, 24, 405-413. 
Table I. Impact of RAE in German U17 First League Clubs $(n=41)$ on success variables.

\begin{tabular}{lccl}
\hline Success indicator & Corr. & $P$ & $\begin{array}{l}\text { Expected difference between team with } \\
\text { largest and smallest RAE }\end{array}$ \\
\hline Rank & 0.328 & 0.036 & 5.5 ranks \\
Points scored & -0.333 & 0.033 & 18.9 points \\
Goals scored & -0.265 & 0.094 & 16.6 goals scored \\
Goals against & .0355 & 0.023 & 20.3 goals against \\
\hline
\end{tabular}

Note: Corr. $=$ Correlation between RAE and Success indicator; $P=$ significance 


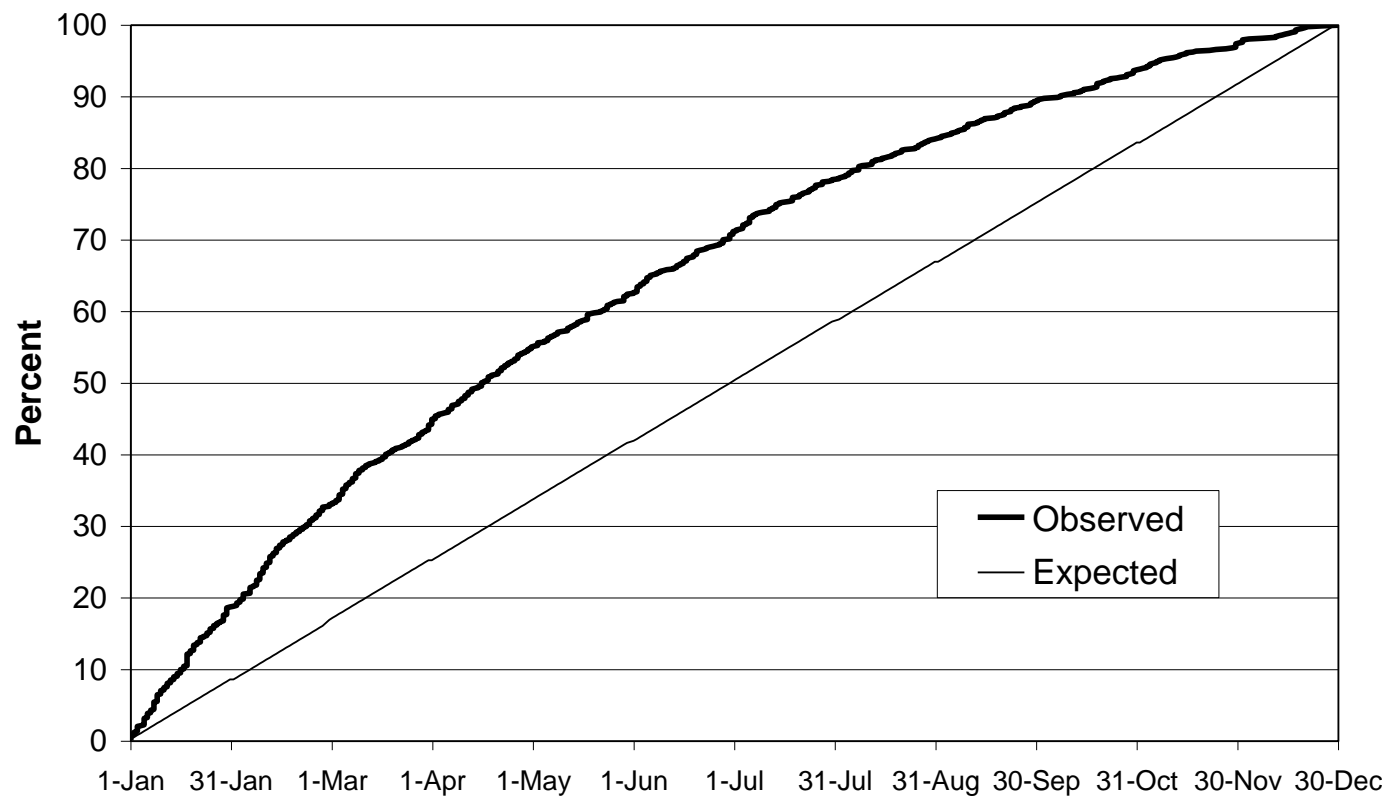

Figure 1. Observed and expected distribution function of birth dates of 911 German U17 2008/09 First League soccer players. The maximum difference (K-S statistics) is $20.71 \%$ at June, $6^{\text {th }}(P<.01)$. 


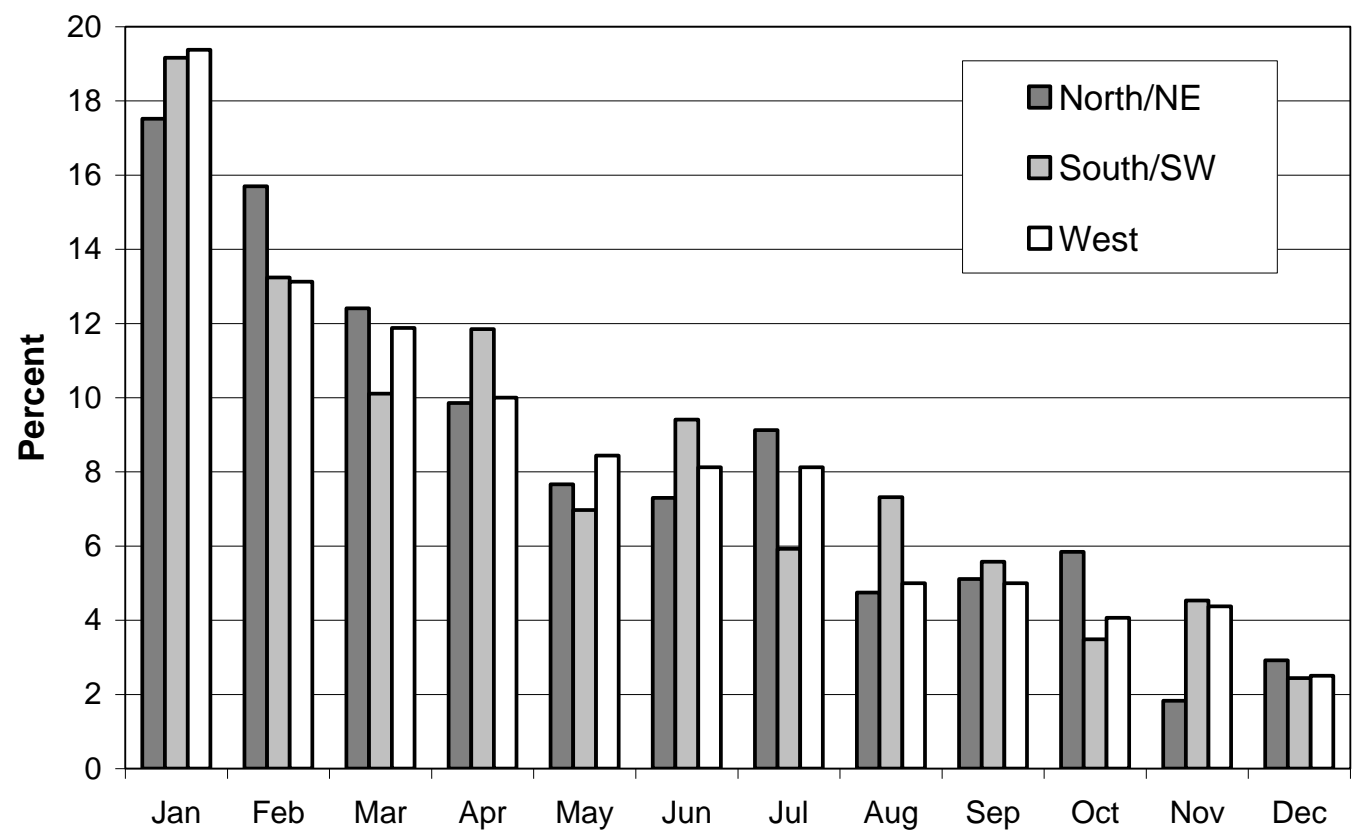

Figure 2. Monthly distribution of birth dates of players from the three German U17 first leagues in the 2008/09 season. 


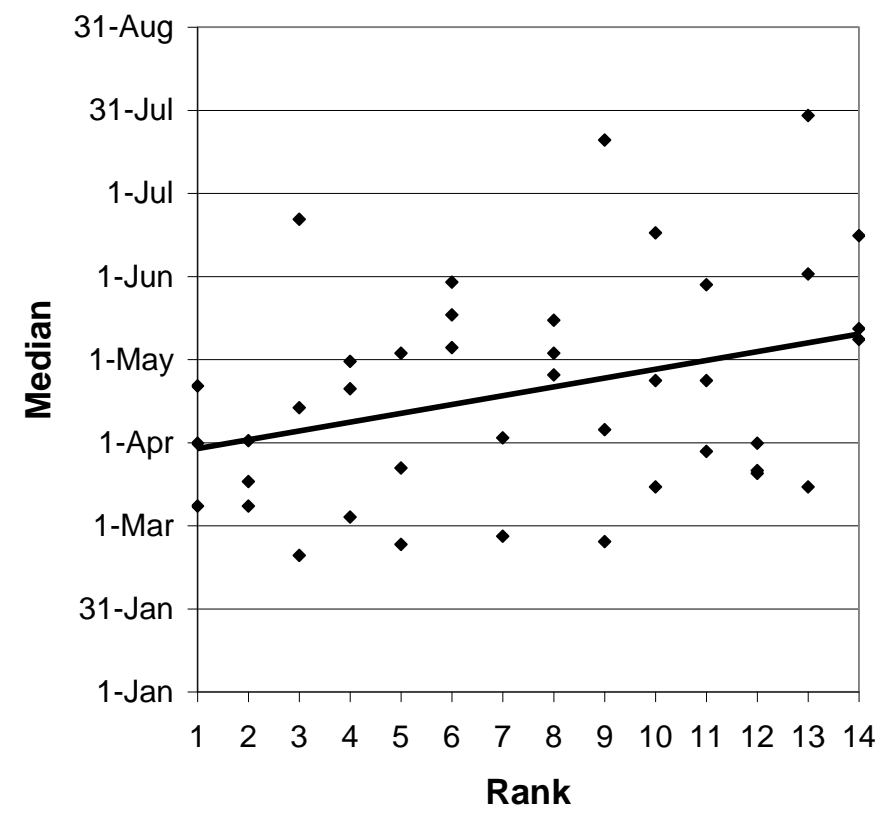

Figure 3. The relation between ranks and medians of birth dates of 41 teams in Germany's three U17 first leagues (Spearman's $\rho=.328, P=.036$ ). 